\title{
Prevalence of serum galectin-1 autoantibodies in seven types of cancer: A potential biomarker
}

\author{
TATSUKI NANAMI $^{1}$, ISAMU HOSHINO ${ }^{2}$, FUMIAKI SHIRATORI ${ }^{1}$, SATOSHI YAJIMA $^{1}$, YOKO OSHIMA ${ }^{1}$, \\ TAKASHI SUZUKI ${ }^{1}$, MASAAKI ITO ${ }^{1}$, TAKAKI HIWASA ${ }^{3}$, AKIKO KUWAJIMA ${ }^{4}$ and HIDEAKI SHIMADA ${ }^{1-3,5}$ \\ ${ }^{1}$ Department of Surgery, School of Medicine, Toho University, Tokyo 143-8541; \\ ${ }^{2}$ Division of Gastroenterological Surgery, Chiba Cancer Center, Chiba 260-8717; \\ ${ }^{3}$ Department of Clinical Oncology, Graduate School of Medicine, Toho University, \\ Tokyo 143-8541; ${ }^{4}$ Medical and Biological Laboratories Co., Ltd., Nagoya, Aichi 460-0008; \\ ${ }^{5}$ Department of Gastroenterological Surgery, Graduate School of Medicine, Toho University, Tokyo 143-8541, Japan
}

Received July 17, 2020; Accepted June 7, 2021

DOI: $10.3892 / \mathrm{mco} .2021 .2341$

\begin{abstract}
Although serum galectin-1 antibodies (s-GAL-1-Abs) have been evaluated in a small number of patients with cancer, a large series of patients with different cancer types have not been reported. The current study evaluated 1,833 patients with esophageal cancer $(n=172)$, gastric cancer $(n=317)$, colorectal cancer $(n=262)$, hepatocellular carcinoma $(n=91)$, prostate cancer $(n=358)$, breast cancer $(n=364)$, lung cancer $(n=269)$ and 72 healthy individuals. s-GAL-1-Abs levels were analyzed using an originally developed ELISA system. A cut-off optical density value was determined as the mean $(0.053)+3$ standard deviations $(0.105)$ of sera from healthy controls. The results revealed that the positive rate of s-GAL-1-Abs in patients with hepatocellular carcinoma (16.7\%) and lung cancer $(13.8 \%)$ were significantly higher compared with the other groups: Esophageal cancer (11.6\%), colorectal cancer (11.5\%), prostate cancer $(7.3 \%)$, gastric cancer $(6.9 \%)$, breast cancer $(6.9 \%)$ and healthy controls (4.2\%). Although the positive rates of s-GAL-1-Abs in different cancer types were relatively low, s-GAL-1-Abs may be useful for patients with hepatocellular carcinoma and lung cancer.
\end{abstract}

Correspondence to: Professor Hideaki Shimada, Department of Gastroenterological Surgery, Graduate School of Medicine, Toho University, 6-11-1 Omori-Nishi, Ota-ku, Tokyo 143-8541, Japan E-mail: hideaki.shimada@med.toho-u.ac.jp

Abbreviations: s-GAL-1-Abs, serum galectin-1 antibodies; HCC, hepatocellular carcinoma; OD, optical density

Key words: galectin-1, serum autoantibody, hepatocellular carcinoma, lung cancer, enzyme-linked immunosorbent assay

\section{Introduction}

Galectin-1, an endogenous lectin found at immune-privileged sites, has a critical role in the regulation of the immune response in the tumor microenvironment (1). Wide varieties of biological phenomena are related to galectins, i.e., development, differentiation, morphogenesis, tumor metastasis, apoptosis, RNA splicing, and immunoregulatory function $(2,3)$. In different cancer types, galectin-1 is abundantly expressed $(4,5)$ and is noted as one of the key players in tumor-mediated immune escape (6). Intra-tumoral high galectin-1 protein expression can be a poor prognostic biomarker in cancers given its immuno-suppressive function (7).

Although galectin-1 protein in cancer tissues is an immune-escape biomarker, its analysis in the serum may be preferable in clinical practice $(8,9)$. Serum galectin-1 levels has been reported to associated with tumor progression and poor prognosis in various types of cancer (10-12). While, serum autoantibodies against various tumor antigens were reported to be useful biomarkers for early detection and for predicting cancer biology $(13,14)$ rather than serum tumor antigen. The prevalence of such autoantibodies (15) should be a fundamental data in designing clinical trials targeting tumor antigens. Previously, we reported that serum galectin-1 autoantibody (s-Gal-1-Abs) is a useful biomarker in hepatocellular carcinoma (16) which might be associated with galectin-1 protein expression. However, there was no data to compare positive rates of s-Gal-1-Abs in various cancer types.

Therefore, in the present study, 1,833 patients with seven different cancer types were evaluated for the presence of s-GAL-1-Abs using the ELISA system. Such fundamental information of s-GAL-1-Abs in a wide range of tumor types may be useful for further clinical studies of immune-therapy or immune-diagnosis.

\section{Materials and methods}

Collection of sera. Before the onset of treatment, sera were obtained from 1,833 patients at Chiba Cancer Center with 
Table I. Characteristics of the patients analyzed in the current study.

\begin{tabular}{lccccc}
\hline & & \multicolumn{3}{c}{ Stage (n) } \\
\cline { 4 - 6 } Type of cancer & Age, years (mean \pm SD) & I & II & III & IV \\
\hline Esophageal cancer & $68.3 \pm 7.7$ & 33 & 38 & 57 & 44 \\
Gastric cancer & $67.0 \pm 10.6$ & 193 & 60 & 21 & 43 \\
Colorectal cancer & $63.4 \pm 10.2$ & 108 & 75 & 41 & 38 \\
Hepatocellular carcinoma & $65.2 \pm 9.0$ & 14 & 40 & 22 & 15 \\
Prostate cancer & $68.5 \pm 7.1$ & 26 & 225 & 60 & 47 \\
Breast cancer & $56.3 \pm 12.5$ & 164 & 157 & 31 & 12 \\
Lung cancer & $66.7 \pm 8.6$ & 98 & 64 & 36 & 71 \\
Healthy control & $37.5 \pm 9.3$ & - & - & - & - \\
\hline
\end{tabular}

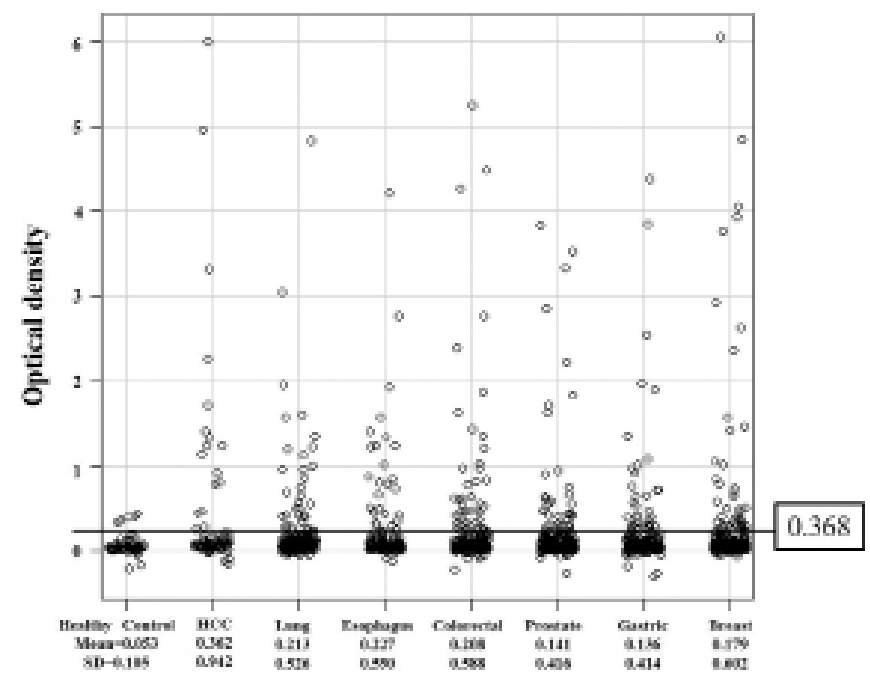

Figure 1. Distribution of serum anti-galectin-1 antibody titers in patients with different types of cancer. A total of 1,833 patients with cancer and 72 healthy controls were evaluated for serum anti-galectin-1 antibody titers. The position of a cut-off value is presented.

different cancer types involving the esophagus $(n=172)$, stomach $(n=317)$, large intestine $(n=262)$, liver $(n=91)$, prostate $(n=358)$, breast $(n=364)$, lung $(n=269)$, and 72 healthy donors. Age and stages were shown in Table I. Each serum sample was centrifuged at 3,000 x $\mathrm{g}$ for $5 \mathrm{~min}$, and the resulting supernatant was stored at $-80^{\circ} \mathrm{C}$ until analyzed at Toho University. We avoided repeated thawing and freezing of the samples. This study was approved by the institutional ethics committee of the Chiba Cancer Center (\#21-26) and the Toho University School of Medicine (\#22-112, \#22-047). Additionally, written informed consent was obtained from all patients. Patient recruitment was conducted from July 2008 to March 2010.

Enzyme-linked immunosorbent assay to detect s-Gal-1-Abs and other conventional tumor markers. Serum samples were analyzed using an enzyme-linked immunosorbent assay, as previously described (16). Purified recombinant GAL-1 protein was coated onto 96-well microtiter plates

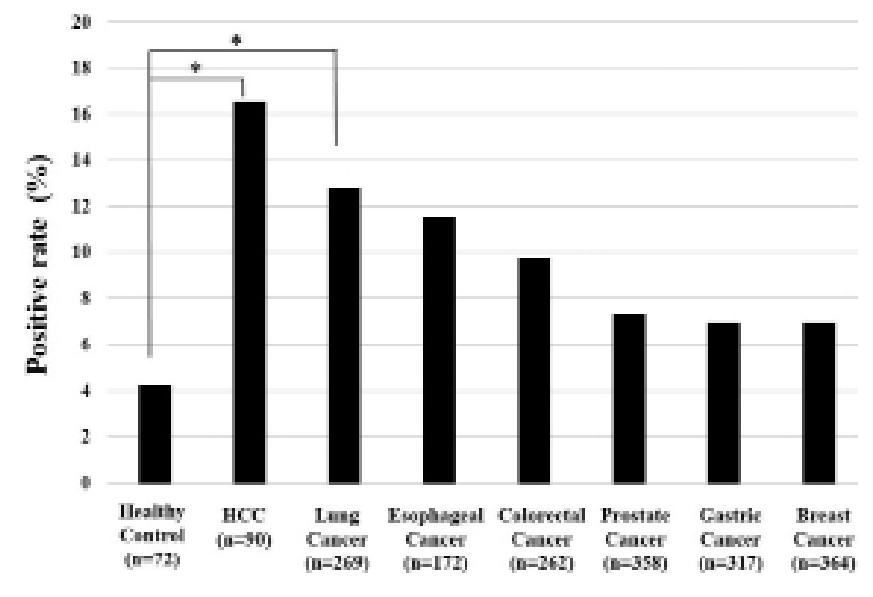

Figure 2. Positive rates of serum galectin-1 antibodies in patients with different types of cancer. ${ }^{*} \mathrm{P}<0.05$ as indicated. HCC, hepatocellular carcinoma.

(Maxisorp; Nunc). The absorbance was measured at $450 \mathrm{~nm}$ using a SUNRISE Microplate Reader (Tecan Japan Co., Ltd.). Gal-1 signals were evaluated by calculating the difference in absorbance between the wells containing galectin-1 and phosphate-buffered saline. Since the antibody titers are displayed in numerical value of absorbance, there is no unit of the protein amount.

Statistical analyses. Fisher's exact (two-sided) probability test was used to determine the differences between the two groups. All statistical analyses were performed using EZR (Saitama Medical Centre, Jichi Medical University; Saitama, Japan) (17), which is a graphical user interface for $\mathrm{R}$ (The R Foundation for Statistical Computing; version 2.13.0). A P-value $<0.05$ was considered to indicate a statistically significant difference.

\section{Results and Discussion}

In the present study, 1,833 patients with different cancer types were evaluated for the presence of s-GAL-1-Abs. Patients with HCC and Lung cancer demonstrated significantly higher positive rates for s-GAL-1-Abs. 


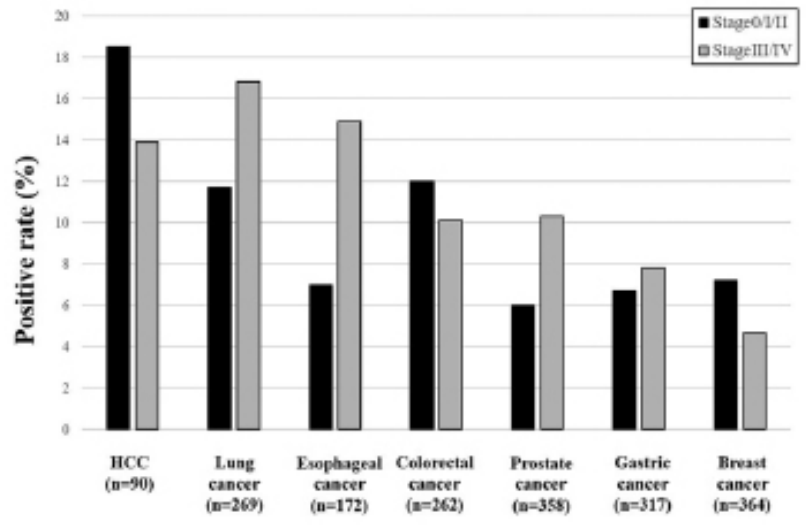

Figure 3. Positive rates of serum anti-galectin-1 antibodies in various types of cancer at tumor stage. HCC, hepatocellular carcinoma.

Serum titers of anti-galectin-1 antibodies. s-GAL-1-Abs levels were divided into two groups; normal optical density (OD) values that were below the border level of 0.368 (calculated as the mean $(0.053)+3$ standard deviations $(0.105)$ of the values in healthy donors) and abnormal, or positive, values that were higher than 0.368 (Fig. 1). Mean OD values for each cancer type were as follows: 0.362 in HCC, 0.213 in lung cancer, 0.227 in esophageal cancer, 0.208 in colorectal cancer, 0.141 in prostate cancer, 0.136 in gastric cancer, and 0.179 in breast cancer.

Positive rates of serum anti-galectin-1 antibodies in various types of tumors. The positive rate of serum anti-GAL-1 antibodies were greater than $10 \%$ in patients with HCC $(16.7 \%)$, lung cancer (13.8\%), esophageal squamous cell carcinoma (11.6\%), and colorectal cancer (11.5\%) (Fig. 2). Other cancer types demonstrated similar positive rates of around 6 to $7 \%$. Although the overall positive rate of s-GAL-1-Abs was not higher than that of conventional tumor markers $(13,18-21)$, it was found to be positive in various cancer types.

The differences of the positive rates of s-GAL-1-Abs among various cancer types might be associated with the positive rates of galectin-1 protein expression in the tumor tissues. Actually, the positive rates of galectin-1 protein expression were $57 \%$ in HCC (22), 51.5\% in lung cancer (23), 30\% in colorectal cancer (24), and $60.6 \%$ in gastric cancer (25). There were no reports on esophageal cancer, prostate cancer, and breast cancer. Although the information of protein expression was limited, there were no association with the positive rate of serum autoantibody.

Unlike conventional secretory tumor markers, autoantibody markers have been reported to be positive even in early stage cancer. However, the positive rates of advanced stages were not always higher than the positive rates of early stages. The exact reason why such paradoxical positive rates was unknow but potential immunosuppression in advanced stages might affect antibody reaction (26-28).

Comparison of positive rates of serum anti-galectin-1 antibodies according to tumor stages. For the comparison of s-GLA-Abs according to the stages, each tumor was divided into Stage 0/I/II and III/VI (Fig. 3). HCC, colorectal cancer, and breast cancer showed higher positive rates in stage $0 / \mathrm{I} / \mathrm{II}$ than in stage III/IV. Lung cancer, esophageal cancer, prostate cancer, and gastric cancer showed higher positive rates in stage III/IV than in stage $0 /$ I/II.

Recent reports showed that galectin-1 targeting therapy may have a potential role to modify anti-cancer immune response $(6,29)$. Galectin-1 vaccination or knockdown therapy could be one of the options for s-GAL-1-Abs positive cancer patients.

Figs. 2 and 3 showed just percentage of positive rates in each cancer types. These percentages were calculated by dividing the positive cases by all cases in Fig. 2. In Fig. 3, the percentages were calculated by dividing the positive cases by total cases at each stage.

In summary, the present study, of the 1,833 patients with seven different cancer types, showed that HCC and lung cancer patients showed relatively higher positive rates of s-GAL-1-Abs than the other cancer types. Based on its low sensitivity, the s-GAL-1-Abs may not be the first choice of a serum marker in other cancers. Such fundamental information of s-GAL-1-Abs in a wide range of tumor types could be a little help for further clinical studies of immune-therapy or immune-diagnosis.

Limitations. Our present study did not include any survival data. Further studies are required in prospective manner to evaluate the prognostic significance and effects on treatment response of serum Galectin-1autoantibodies.

\section{Acknowledgments}

The authors would like to acknowledge Ms. Seiko Ohtsuka (Research Secretary of Toho University Hospital) for sampling and preparing the database for this study.

\section{Funding}

The current study was supported by a Grant-in-Aid for Scientific Research from the Ministry of Education, Culture, Sports, Science and Technology of Japan (grant nos. 24591961 and 21591717).

\section{Availability of data and materials}

The datasets used and/or analyzed in the present study are available from the corresponding author on reasonable request.

\section{Authors' contributions}

TN, IH, MI and HS conceived and designed the current study. SY, YO, TS, and FS analyzed the data. AK developed the ELISA system. TN, TH and HS analyzed patient data and drafted the manuscript. HS and TN confirm the authenticity of all the raw data. All authors have read and approved the final manuscript.

\section{Ethics approval and consent to participate}

The current study was approved by the institutional Ethics Committees of the Chiba Cancer Center (approval no. \#21-26) and the Toho University School of Medicine (approval 
nos. \#22-112 and \#22-047). Additionally, written informed consent was obtained from all patients.

\section{Patient consent for publication}

Not applicable.

\section{Competing interests}

HS received a research grant from Medical \& Biological Laboratories Co., Ltd.. The other authors declare that they have no competing interests.

\section{References}

1. Martínez-Bosch N and Navarro P: Galectins in the tumor microenvironment: Focus on galectin-1. Adv Exp Med Biol 1259: 17-38, 2020.

2. Elola MT, Chiesa ME, Alberti AF, Mordoh J and Fink NE: Galectin-1 receptors in different cell types. J Biomed Sci 12 $13-29,2005$

3. Thijssen VL, Heusschen R, Caers J and Griffioen AW: Galectin expression in cancer diagnosis and prognosis: A systematic review. Biochim Biophys Acta 1855: 235-247, 2015.

4. Chung LY, Tang SJ, Sun GH, Chou TY, Yeh TS, Yu SL and Sun KH: Galectin-1 promotes lung cancer progression and chemoresistance by upregulating p38 MAPK, ERK, and cyclooxygenase-2. Clin Cancer Res 18: 4037-4047, 2012.

5. Holst JM, Ludvigsen M, Hamilton-Dutoit SJ, Bendix K, Plesner TL, Nørgaard P, Møller MB, Steiniche T, Rabinovich GA, d'Amore F and Pedersen MB: High intra-tumoural galectin-1 expression predicts adverse outcome in $\mathrm{ALK}^{-} \mathrm{ALCL}$ and $\mathrm{CD} 30^{+}$ PTCL-NOS. Hematol Oncol 38: 59-66, 2020.

6. Verschuere T, Toelen J, Maes W, Poirier F, Boon L, Tousseyn T, Mathivet T, Gerhardt H, Mathieu V, Kiss R, et al: Glioma-derived galectin-1 regulates innate and adaptive antitumor immunity. Int J Cancer 134: 873-884, 2014.

7. Gajiwala S, Torgeson A, Garrido-Laguna I, Kinsey C and Lloyd S: Combination immunotherapy and radiation therapy strategies for pancreatic cancer-targeting multiple steps in the cancer immunity cycle. J Gastrointest Oncol 9: 1014-1026, 2018.

8. Petrovic S, Radosavljevic GD, Pantic J, Jovanovic I, Jankovic N and Arsenijevic N: Circulating and tissue galectin-1 and galectin-3 in colorectal carcinoma: Association with clinicopathological parameters, serum CEA, IL-17 and IL23. J BUON 21: 941-949, 2016

9. Chen L, Yao Y, Sun L, Zhou J, Liu J, Wang J, Li J and Tang J: Clinical implication of the serum galectin-1 expression in epithelial ovarian cancer patients. J Ovarian Res 8: 78, 2015.

10. Chen K, Cai Y, Zhang M, Wu Z and Wu Y: Both serum and tissue galectin-1 levels are associated with adverse clinical features in neuroblastoma. Pediatr Blood Cancer 65: e27229, 2018.

11. Gurel Cayir E, Demir L, Varol U, Atahan MK, Salman T, Oflazoglu U, Yildiz Y, Taskaynatan H, Saray S, Kucukzeybek Y, et al: Preliminary study of serum galectin-1 in breast cancer carcinogenesis [Izmir Oncology Group (IZOG) study]. J BUON 25: 675-680, 2020.

12. Wu KL, Chen HH, Pen CT, Yeh WL, Huang EY, Hsiao CC and Yang KD: Circulating galectin-1 and 90K/Mac-2BP correlated with the tumor stages of patients with colorectal cancer. Biomed Res Int 2015: 306964, 2015.

13. Shimada H, Ochiai T and Nomura F; Japan p53 Antibody Research Group: Titration of serum p53 antibodies in 1,085 patients with various types of malignant tumors: A multi-institutional analysis by the Japan p53 antibody research group. Cancer 97: 682-689, 2003.

14. Shimada H, Takeda A, Arima M, Okazumi S, Matsubara H, Nabeya Y, Funami Y, Hayashi H, Gunji Y, Suzuki T, et al: Serum p53 antibody is a useful tumor marker in superficial esophageal squamous cell carcinoma. Cancer 89: 1677-1683, 2000.
15. Oshima Y, Shimada H, Yajima S, Nanami T, Matsushita K, Nomura F, Kainuma O, Takiguchi N, Soda H, Ueda T, et al: NY-ESO-1 autoantibody as a tumor-specific biomarker for esophageal cancer: Screening in 1969 patients with various cancers. J Gastroenterol 51: 30-34, 2016.

16. Shiratori F, Shimada H, Nagata M, Kubota Y, Otsuka Y and Kaneko H: Serum galectin-1 autoantibodies in patients with hepatocellular carcinoma. Toho J Med 2: 67-72, 2016.

17. Kanda Y: Investigation of the freely available easy-to-use software 'EZR' for medical statistics. Bone Marrow Transplant 48: 452-458, 2013.

18. Ushigome M, Shimada H, Miura Y, Yoshida K, Kaneko T, Koda T, Nagashima Y, Suzuki T, Kagami S and Funahashi K: Changing pattern of tumor markers in recurrent colorectal cancer patients before surgery to recurrence: Serum p53 antibodies, CA19-9 and CEA. Int J Clin Oncol 25: 622-632, 2020.

19. Shimada H, Noie T, Ohashi M, Oba K and Takahashi Y: Clinical significance of serum tumor markers for gastric cancer: A systematic review of literature by the task force of the Japanese gastric cancer association. Gastric Cancer 17: 26-33, 2014.

20. Sengupta S and Parikh ND: Biomarker development for hepatocellular carcinoma early detection: Current and future perspectives. Hepat Oncol 4: 111-122, 2017.

21. Nakamura $\mathrm{H}$ and Nishimura T: History, molecular features, and clinical importance of conventional serum biomarkers in lung cancer. Surg Today 47: 1037-1059, 2017.

22. Yeh CC, Hsu CH, Shao YY, Ho WC, Tsai MH, Freg WC and Chow LP: Integrated stable isotope labeling by aminoacids in cell culture (SILAC) and isobaric tags for relative and absolute quantitation (iTRAQ) quantitative proteomic analysis identifies galectin-1 as a potential biomarker for predicting sorafenib resistance in liver cancer. Mol Cell Proteomics 14: 1527-1545, 2015.

23. Carlini MJ, Roitman P, Nuñez M, Pallotta MG, Boggio G, Smith D, Salatino M, Joffé ED, Rabinovich GA and Puricelli LI: Clinical relevance of galectin-1 expression in non-small cell lung cancer patients. Lung Cancer 84: 73-78, 2014.

24. Nagy N, Legendre H, Engels O, André S, Kaltner H, Wasano K, Zick Y, Pector JC, Decaestecker C, Gabius HJ, et al: Refined prognostic evaluation in colon carcinoma using immunohistochemical galectin fingerprinting. Cancer 97: 1849-1858, 2003.

25. He XJ, Tao HQ, Hu ZM, Ma YY, Xu J, Wang HJ, Xia YJ, Li 1, Fei BY, Li YQ and Chen JZ: Expression of galectin-1 in carcinoma-associated fibroblasts promotes gastric cancer cell invasion through upregulation of integrin $\beta 1$. Cancer Sci 105: 1402-1410, 2014.

26. Okada R, Otsuka Y, Wakabayashi T, Shinoda M, Aoki T, Murakami M, Arizumi S, Yamamoto M, Aramaki O, Takayama T, et al: Six autoantibodies as potential serum biomarkers of hepatocellular carcinoma: A prospective multicenter study. Int J Cancer 47: 2578-2586, 2020.

27. Takashi S, Satoshi Y, Akihiko O, Naoya Y, Yusuke T, Kentaro M, Yu O, Yasuaki N, Koichi Y, Takashi F, et al: Clinical impact of preoperative serum p53 antibody titers in 1,487 patients with surgically treated esophageal squamous cell carcinoma: A multi-institutional study. Esophagus 18: 65-71, 2021.

28. Shimada H, Nagata M, Nabeya Y, Yajima S, Oshima Y and Itami M: Paradoxical changing of serum p53 antibody titers during chemotherapy for a stage IV esophageal squamous cell carcinoma. Int Cancer Conf J 3: 232-236, 2014.

29. Van Woensel M, Mathivet T, Wauthoz N, Rosière R, Garg AD, Agostinis P, Mathieu V, Kiss R, Lefranc F, Boon L, et al: Sensitization of glioblastoma tumor micro-environment to chemo- and immunotherapy by galectin-1 intranasal knock-down strategy. Sci Rep 7: 1217, 2017.

This work is licensed under a Creative Commons Attribution-NonCommercial-NoDerivatives 4.0 International (CC BY-NC-ND 4.0) License. 